Pacific Journal of Mathematic 


\title{
A NOTE ON A PAPER BY L. C. YOUNG
}

\author{
F. W. GEHRIN G
}

1. Introduction. Suppose that $f(x)$ is a real- or complex-valued function defined for all real $x$. For $0 \leq \alpha \leq 1$, we define the $\alpha$-variation of $f(x)$ over $a \leq x \leq b$ as the least upper bound of the sums

$$
\left\{\sum|\Delta f|^{1 / a}\right\}^{a}
$$

taken over all finite subdivisions of $a \leq x \leq b$. (When $\alpha=0$, we denote by the above sum simply the maximum $|\Delta f|$.) We say that $f(x)$ is in $W_{\alpha}$ if it has finite $a$-variation over the interval $0 \leq x \leq 1$. L. C. Young has proved the following result.

Theorem 1. (See [2, Theorem 4.2].) Suppose that $0<\beta<1$ and that $f(x)$, with period 1 , satisfies the condition

$$
\int_{0}^{1}|f\{\phi(t+h)\}-f\{\phi(t)\}| d t \leq h^{\beta}
$$

for every monotone function $\phi(t)$ such that

$$
\phi(t+1)=\phi(t)+1
$$

for all t. Then $f(x)$ is in $\mathbb{W}_{\alpha}^{\prime}$ for each $\alpha<\beta$.

Young's argument does not suggest whether we can assert that $f(x)$ is in $W_{\beta}$. We present here an elementary proof for Theorem 1 and an example to show that this result is the best possible one in this direction.

2. Lemma. We require the following:

LEMma 2. Suppose that $a_{1}, a_{2}, \cdots, a_{N}$ and $b_{1}, b_{2}, \cdots, b_{N}$ are two sets of nonnegative numbers such that $a_{1} \geq a_{2} \geq \cdots \geq a_{N}$ and such that

Received July 2, 1953.

$$
\sum_{\nu=1}^{n} a_{\nu} \leq \sum_{\nu=1}^{n} b_{\nu}
$$

Pacific J. Math. 5 (1955), 67-72 
for $n=1, \cdots, N$. Then for $p>1$,

$$
\sum_{\nu=1}^{n} a_{\nu}^{p} \leq \sum_{\nu=1}^{n} b_{\nu}^{p}
$$

for $n=1, \cdots, N$.

Let

$$
S_{n}=\sum_{\nu=1}^{n} a_{\nu} \text { and } T_{n}=\sum_{\nu=1}^{n} b_{\nu} .
$$

With Abel's identity and Hölder's inequality, we have

$$
\begin{aligned}
\sum_{\nu=1}^{n} a_{\nu}^{p} & =\sum_{\nu=1}^{n} a_{\nu} a_{\nu}^{p-1} \\
& =S_{1}\left(a_{1}^{p-1}-a_{2}^{p-1}\right)+\cdots+S_{n-1}\left(a_{n-1}^{p-1}-a_{n}^{p-1}\right)+S_{n} a_{n}^{p-1} \\
& \leq T_{1}\left(a_{1}^{p-1}-a_{2}^{p-1}\right)+\cdots+T_{n-1}\left(a_{n-1}^{p-1}-a_{n}^{p-1}\right)+T_{n} a_{n}^{p-1} \\
& =\sum_{\nu=1}^{n} b_{\nu} a_{\nu}^{p-1}, \\
& \leq\left\{\sum_{\nu=1}^{n} b_{\nu}^{p}\right\}^{1 / p}\left\{\sum_{\nu=1}^{n} a_{\nu}^{p}\right\}^{(p-1) / p},
\end{aligned}
$$

from which the lemma follows.

3. Proof of Theorem 1. For a subdivision $0=x_{0}<x_{1}<\cdots<x_{N}=1$, consider the numbers

$$
\left|f\left(x_{1}\right)-f\left(x_{0}\right)\right|,\left|f\left(x_{2}\right)-f\left(x_{1}\right)\right|, \ldots,\left|f\left(x_{N}\right)-f\left(x_{N-1}\right)\right|,
$$

and label this set $a_{1}, a_{2}, \cdots, a_{N}$ so that $a_{1} \geq a_{2} \geq \cdots \geq a_{N}$. We say that the two points $\xi^{\prime}$ and $\xi^{\prime \prime}$ are associated with $a_{n}$ if they are the two points of the subdivision for which

$$
a_{n}=\left|f\left(\xi^{\prime \prime}\right)-f\left(\xi^{\prime}\right)\right|
$$

and, fixing $n$, we consider the union of points associated with $a_{1}, a_{2}, \cdots, a_{n}$. Labeling these $\xi_{1}<\xi_{2}<\cdots<\xi_{m_{n}}$, we define 


$$
\phi(t)=\xi_{\nu} \text { for } \frac{\nu-1}{m_{n}} \leq t<\frac{\nu}{m_{n}} \quad\left(\nu=1, \cdots, m_{n}\right),
$$

and we extend this function so that

$$
\phi(t+1)=\phi(t)+1
$$

Now $m_{n} \leq 2 n$ and, if $0<h<1 / m_{n}$,

$$
\begin{aligned}
h \sum_{\nu=t}^{n} a_{\nu} & \leq h \sum_{\nu=2}^{m_{n}}\left|f\left(\xi_{\nu}\right)-f\left(\xi_{\nu-1}\right)\right| \\
& \leq \int_{0}^{1}|f\{\phi(t+h)\}-f\{\phi(t)\}| d t \leq h^{\beta}
\end{aligned}
$$

Letting $h$ approach $1 / m_{n}$, we have

$$
\sum_{\nu=1}^{n} a_{\nu} \leq m_{n}^{1-\beta} \leq(2 n)^{1-\beta}
$$

for $n=1, \cdots, N$. Finally selecting $b_{1}, b_{2}, \cdots, b_{N}$ so that

$$
\sum_{\nu=1}^{n} b_{\nu}=(2 n)^{1-\beta}
$$

we have

$$
b_{1}=2^{1-\beta} \text { and } b_{n}<2^{1-\beta}(n-1)^{-\beta} \quad \text { for } n>1 \text {, }
$$

and applying I emma 2 we conclude that

$$
\left\{\sum_{n=1}^{N}\left|\Delta_{n} f\right|^{1 / \alpha}\right\}^{\alpha} \leq\left\{\sum_{n=1}^{N} b_{n}^{1 / \alpha}\right\}^{\alpha}<2\left\{\sum_{n=1}^{\infty} n^{-\beta / \alpha}\right\}^{\alpha}
$$

This completes the proof.

4. Further results. We now show that Theorem 1 is best possible.

THEOREM 3. Suppose that $0<\beta<\gamma \leq 1$. There exists a function $f(x)$, with period 1 , which is not in $\mathbb{W}_{\beta}$ and which satisfies the condition 


$$
\left\{\int_{0}^{1}|f\{\phi(t+h)\}-f\{\phi(t)\}|^{1 / \gamma}\right\}^{\gamma} \leq h^{\beta}
$$

for every monotone function $\phi(t)$ such that

$$
\phi(t+1)=\phi(t)+1 .
$$

Consider two increasing sequences, $\left\{x_{n}\right\}$ and $\left\{y_{n}\right\}$, such that

$$
x_{1}<y_{1}<x_{2}<\cdots<x_{n}<y_{n}<x_{n+1}<\cdots<x_{1}+1 .
$$

Define the function

$$
g(x)= \begin{cases}n^{-\beta} & \text { for } x_{n}<x<y_{n}, \\ 0 & \text { everywhere else in } x_{1} \leq x<x_{1}+1,\end{cases}
$$

and extend $g(x)$ to have period 1 .

Lemma 4. Suppose that $0<\beta<\gamma \leq 1$. The function $g(x)$ defined above satisfies the condition

$$
\left\{\int_{0}^{1}|g(x+h)-g(x)|^{1 / \gamma} d x\right\}^{\gamma} \leq\left(\frac{2 \gamma}{\gamma-\beta}\right)^{\gamma} h^{\beta} \quad(h \geq 0) .
$$

Fix $h$ in the range $0<h \leq 1 / 2$, and consider the finite sequence,

$$
\xi_{0}<\xi_{1}<\cdots<\xi_{N}=\xi_{0}+1
$$

defined as follows.

A. Let $\xi_{0}=x_{1}-h$.

B. Suppose that $\xi_{0}<\xi_{1}<\cdots<\xi_{n-1}<\xi_{0}+1$ have been defined. Let $\xi_{n}=\operatorname{Max}\left\{\xi_{n-1}+2 h, y_{n}\right\}$ if this does not exceed $\xi_{0}+1$. Otherwise let $\xi_{n}=\xi_{0}+1$.

It is not difficult to show that

$$
\int_{\xi_{n-1}}^{\xi_{n}}|g(x+h)-g(x)|^{1 / \gamma} d x \leq 2 h n^{-\beta / \gamma}
$$

for $n=1, \cdots, N$. Since $\xi_{n}-\xi_{n-1} \geq 2 h$ for $n=1, \cdots, N-1$, we have $N h<1$ and 


$$
\begin{aligned}
\int_{0}^{1}|\Delta g|^{1 / \gamma} d x & =\sum_{n=1}^{N} \int_{\xi_{n-1}}^{\xi_{n}}|\Delta g|^{1 / \gamma} d x \leq 2 h \sum_{n=1}^{N} n^{-\beta / \gamma}, \\
& <\frac{2}{1-\beta / \gamma} h N^{1-\beta / \gamma}<\frac{2 \gamma}{\gamma-\beta} h^{\beta / \gamma} .
\end{aligned}
$$

This completes the proof of Lemma 4.

Take any strictly increasing continuous function $\phi(t)$ such that

$$
\phi(t+1)=\phi(t)+1 \text {. }
$$

If $\phi^{-1}$ is the inverse function, and

$$
u_{n}=\phi^{-1}\left(x_{n}\right) \text { and } v_{n}=\phi^{-1}\left(y_{n}\right) \text {, }
$$

then $u_{1}<v_{1}<u_{2}<\cdots<u_{n}<v_{n}<u_{n+1}<\cdots<u_{1}+1$ and

$$
g\{\phi(t)\}= \begin{cases}n^{-\beta} & \text { for } u_{n}<t<v_{n}, \\ 0 & \text { everywhere else in } u_{1} \leq t<u_{1}+1 .\end{cases}
$$

Now $g\{\phi(t)\}$ has period 1 in $t$, and, by Lemma 4,

$$
\left\{\int_{0}^{1}|g\{\phi(t+h)\}-g\{\phi(t)\}|^{1 / \gamma} d t\right\}^{\gamma} \leq\left(\frac{2 \gamma}{\gamma-\beta}\right)^{\gamma} h^{\beta} \quad(h \geq 0) .
$$

The Lebesgue limit theorem allows us to conclude this holds for all nondecreasing $\phi(t)$ such that

$$
\phi(t+1)=\phi(t)+1
$$

To complete the proof of Theorem 3, observe that $g(x)$ is not in $W_{\beta}$ and let

$$
f(x)=\left(\frac{\gamma-\beta}{2 \gamma}\right)^{\gamma} g(x) .
$$

In the proof of Theorem 3 , the fact that $\beta<\gamma$ plays an important role. We have a different situation when $\beta=\gamma$.

ТНЕОВЕм 5. Suppose that $0 \leq \beta \leq 1$ and that $f(x)$ is measurable and realvalued with period 1 . The $\beta$-variation of $f(x)$ over any interval of length 1 does not exceed 1 if and only if 


$$
\left\{\int_{0}^{1}|f\{\phi(t+h)\}-f\{\phi(t)\}|^{1 / \beta} d t\right\}^{\beta} \leq h^{\beta}
$$

for each monotone function $\phi(t)$ such that $\phi(t+1)=\phi(t)+1$.

For the sufficiency, let $x_{0}<\cdots<x_{N}=x_{0}+1$ be a subdivision of some interval of length 1. Define the function

$$
\phi(t)=x_{n}, \quad \frac{n}{N} \leq t<\frac{n+1}{N} \quad(n=0, \cdots, N-1),
$$

and extend $\phi(t)$ so that

$$
\phi(t+1)=\phi(t)+1
$$

for $0<h<1 / N$ we get

$$
\left\{\sum_{n=1}^{N}\left|\Delta_{n} f\right|^{1 / \beta}\right\}^{\beta} \leq\left\{\frac{1}{h} \int_{0}^{1}|f\{\phi(t+h)\}-f\{\phi(t)\}|^{1 / \beta} d t\right\}^{\beta} \leq 1 .
$$

For the necessity, we see that the $\beta$-variation for $f\{\phi(t)\}$ over any interval of length 1 does not exceed 1 , and we can apply the following:

Thе овем 6. (See [1, Theorem 1.3.3].) Suppose that $0 \leq \beta \leq 1$, that $f(x)$ is measurable and real-valued with period 1 , and that the $\beta$-variation of $f(x)$ over any interval of length 1 does not exceed 1 . Then

$$
\left\{\int_{0}^{1}|f(x+h)-f(x)|^{1 / \beta} d x\right\}^{\beta} \leq h^{\beta} \quad(h \geq 0) .
$$

\section{Peferences}

1. F. W. Gehring, A study of a-variation, I, Trans. Amer. Math. Soc. 76 (1954), $420-443$.

2. L. C. Young, Inequalities connected with bounded $p$-th power variation in the Wiener sense and with integrated Lipschitz conditions, Proc. London Math. Soc. (2) 43 (1937), 449- 467.

Peterhouse, Cambridge, England

HaRVARD UNiverSity, CAMBridge, MASS. 



\section{Pacific Journal of Mathematics}

\section{Vol. 5, No. $1 \quad$ September, 1955}

Frank Herbert Brownell, III, Flows and noncommuting projections on

Hilbert space ................................... 1

H. E. Chrestenson, A class of generalized Walsh functions ............. 17

Jean Bronfenbrenner Crockett and Herman Chernoff, Gradient methods of maximization ................................... 33

Nathan Jacob Fine, On groups of orthonormal functions. I .......... 51

Nathan Jacob Fine, On groups of orthonormal functions. II ............ 61

Frederick William Gehring, A note on a paper by L. C. Young .......... 67

Joachim Lambek and Leo Moser, On the distribution of Pythagorean

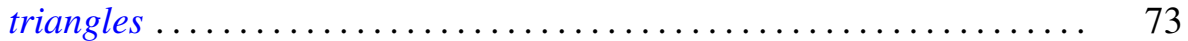

Roy Edwin Wild, On the number of primitive Pythagorean triangles with

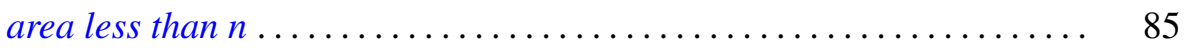

R. Sherman Lehman, Approximation of improper integrals by sums over multiples of irrational numbers ........................ 93

Emma Lehmer, On the number of solutions of $u^{k}+D \equiv w^{2}(\bmod p) \ldots 103$

Robert Delmer Stalley, A modified Schnirelmann density............... 119

Richard Allan Moore, The behavior of solutions of a linear differential equation of second order............................. 125

William M. Whyburn, A nonlinear boundary value problem for second order differential systems. 